\title{
Numerical investigation of the hydrocyclone vortex finder depth on separation efficiency
}

\author{
Lesiba Mokonyama ${ }^{1, *}$, Thokozani Justin Kunene ${ }^{1}$, and Lagouge Kwanda Tartibu ${ }^{1}$
}

${ }^{1}$ University of Johannesburg, Mechanical \& Industrial Engineering Technology Department, Doornfontein Campus, Johannesburg, South Africa

\begin{abstract}
Hydrocyclones are devices used in numerous chemicals, food, and mineral-related industrial sectors for the separation of fine particles. A $\mathrm{d} 50 \mathrm{~mm}$ hydrocyclone was modelled with the use of the Computational fluid dynamics (CFD) simulation, ANSYS ${ }^{R}$ Fluent 2021 R1. The vortex finder depth was varied from $20 \mathrm{~mm}, 30 \mathrm{~mm}$, and $35 \mathrm{~mm}$ to observe the effects of pressure drop and separation efficiency from a varied vortex finder depth and characteristics of the air core. The numerical methods validated the results observed from different parameters such as volume fraction characteristics based on CFD simulations. The tangential and axial velocities increased as the vortex finder length increased. It was found that as the depth of the vortex finder is increased, particle re-entrainment time in the underflow stream increases, and separation efficiency improved.
\end{abstract}

Keywords:

Computational Fluid Dynamics, Hydrocyclone, Pressure Drop, Vortex Finder

\section{Introduction}

Despite its widespread use in chemical and mineral processing, determining the geometry and operating conditions of a hydrocyclone for a given application in the separation or classification of solids is still based on empirical equations or simplified theories of flow and separation mechanism within the hydrocyclone. Hydrocyclones are frequently utilized in various separation applications because of their inexpensive operating and maintenance costs, high throughput, and small footprint [1]. Various operational and structural elements determine the flow field in a hydrocyclone, which is characterized by a complicated threedimensional whirling flow with an air core. The majority of operational factors are feed rate and slurry concentration is two factors to consider. The structural parameters are feed inlet form, vortex finder form, overflow outlet diameter, underflow outlet diameter, and cone angle. Martínez et al [2] used a stroboscope to measure the detailed velocity profiles in a hydrocyclone. Simulations with a hydrocyclone at a constant flow rate, overflow-tothroughput ratios, entries, and vortex finder diameters were carried out to understand the dependence of hydrocyclone performance on feed. Roping is a phenomenon that is yet primarily unknown. Because of variations in cut size and flow split, it has significant operational implications. According to Plitt and Booth [3], it is more crucial since the highest separating efficiency is attained close to the rope discharge regime. They have created response surfaces for separation efficiency as a function of classifier orifices and feed rate.

${ }^{1}$ Lesiba Mokonyama. Lesiba.k.mokonyama@gmail.com 
Roping has been chiefly investigated in stand-alone hydro cyclones. In continuous closed grinding circuits, only a tiny amount of research has been done [3].

The form of the underflow, which is controlled by the feed circumstances, has a significant impact on hydrocyclone separation. Rope discharge with high feed solids concentrations usually results in a crude product with higher solids content and fewer particles. Spray discharge with low input solids concentration, on the other hand, results in more significant solids recovery and smaller cut sizes. As a result, ideal overall conditions may be expected at the rope/spray discharge changeover point [4][5]. Even though the impact of solids content in the feed on hydrocyclone separation efficiency has long been recognized, comprehensive study on the change in pressure drop across the hydrocyclone and the latter's separation characteristics has been absent. If all other operational parameters remain constant, an increase in feed concentration will usually result in coarser cut size, decreased separation sharpness, and increased pressure drop. Aside from the particles impeding each other's radial motion, the apex valve is limited capacity and variations in the flow field within the hydrocyclone cause more particles to be entrained by the overflow [6][7].

Raesi and Mahddahian [8] investigated air inside the air-injected deoiling hydrocyclone. Thy found that an air core forms at the centre of the hydrocyclone due to the lower density of air compared to oil and water. The volume fraction of air in the hydrocyclone's core approaches one. The majority of the air fed into the hydrocyclone escapes through the overflow. As a result, air bubble separation efficiency is 97.4 percent. There is always a need to eliminate or drastically reduce the air core formed inside the hydrocyclone to improve the separation efficiency. Sripriya et al [9] investigated a new hydrocyclone was constructed with the insertion of a solid rod at the core region of the conical section. By doing so, the air core could be successfully eliminated, and the hydrocyclone's performance could be improved. The lowering of radial and axial components of velocity and turbulence in the area near the vortex finder's entrance may cause this effect. As a result, the flow field characteristics inside the hydrocyclone without an air core become more separation-friendly. Critically to the performance in terms of air core reduction in the hydrocyclone are the feed conditions. Hararah et al [10] determined through their experimental results that when the feed-solids content increased, the air-core diameter shrank, which was supported by the theoretical consideration. Finally, at a critical high feed-solids level, where rope discharge of the underflow was reached, the air core vanished. The air-core diameter was associated with the underflow spray angle at low feed-solids concentration. We have also our findings can be used not only to build a thorough picture of the vortex finder's action, but also to create and control hydrocyclone's performances.

\section{Model description}

\subsection{Hydrocyclone geometry and grid generation}

The hydrocyclone's geometry was utilized in this work; numerical and experimental models are used, as seen in Figure 1. It is a standard hydrocyclone with specified dimensions for sorting mineral particles. 

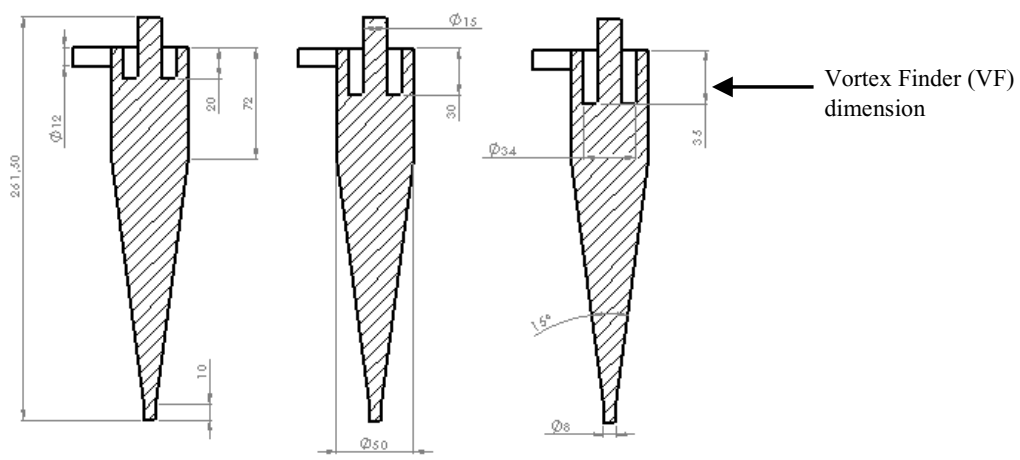

Fig. 1. Geometry of hydrocyclone of base case (unit: $\mathrm{mm}$ ) representing three vortex finder (VF) lengths, $20 \mathrm{~mm}, 30 \mathrm{~mm}$ and $35 \mathrm{~mm}$, respectively

Table 1 lists the parameters investigated in this study, which include air core investigation and flow structures based on the base case; variable parameters such as feed rate, cone angle, underflow outlet diameter, and overflow outlet diameter are investigated to study the influence of different parameters on the flow field inside the hydrocyclone.

Table 1: Geometry of hydrocyclone of base case (unit: $\mathrm{mm}$ )

\begin{tabular}{lcc}
\hline \multicolumn{1}{c}{ Parameter } & Base & Variable \\
\hline Feed Rate $(\mathrm{m} / \mathrm{s})$ & 3 & - \\
Cone Angle $\left({ }^{\circ}\right)$ & 15 & - \\
Underflow Diameter $(\mathrm{mm})$ & 8 & - \\
Overflow Diameter $(\mathrm{mm})$ & 15 & - \\
Vortex finder Depth $(\mathrm{mm})$ & 20 & $20,30,35$ \\
\hline
\end{tabular}

Figure 2 shows a mesh generated using the tetrahedron with seven inflation layers at the first thickness of $10^{-5}$ leading to the wall plus value of $y+=5$. The ANSYS ${ }^{\circledR}$ Fluent $2021 \mathrm{R} 1$ (student version) has a limit of 510,000 elements. We meshed close to the limit of and archived 478560 elements. These elements were accepted to be valid for the model based of the limitation of the commercial code.
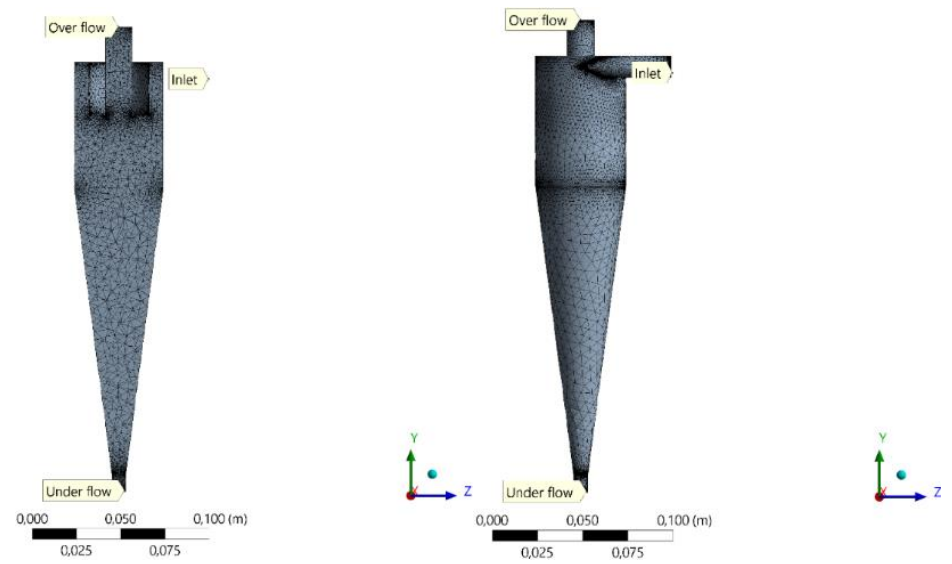

Fig. 2. Mesh density of tetrahedral elements for a computational domain a hydrocyclone. 


\subsection{Volume of fluid (VOF) equations}

The governing equation of volume fraction in VOF model can be expressed by Li et al. [11] as:

$\frac{\partial \alpha_{q}}{\partial t}+\vec{u} \cdot \nabla \alpha_{q}=0$

Where $\alpha_{\mathrm{q}}$ denotes the volume fraction of the, i-phase fluid. When the whole space is full of a same phase, $\alpha_{q}=1$; when the whole space is full of two phases, the sum of the volume fractions of two phases equal to 1 .

\subsection{Turbulence model}

\subsubsection{Shear Stress Transport (SST) model}

Menter [12] developed the SST $k$-turbulence model, and the governing equations of the flow field, comprising the continuity equation, momentum equations, and transport equations of $k$ and $\omega$, can be expressed as follows.

The SST turbulence model comprises two equations: one for $k$, the specific turbulent kinetic energy $\left(\mathrm{m}^{2} \mathrm{~s}^{-2}\right)$

$\frac{\partial(\rho k)}{\partial t}+\frac{\partial\left(\rho u_{j} k\right)}{\partial x_{j}}=P-\beta^{*} \rho \omega k+\frac{\partial}{\partial x_{j}}\left[\left(\mu+\sigma_{k} \mu_{t}\right) \frac{\partial k}{\partial x_{j}}\right]$

And one for $\omega$, the specific turbulent dissipation rate (or specific turbulent frequency) $\left(\mathrm{s}^{-1}\right)$

$$
\frac{\partial(\rho \omega)}{\partial t}+\frac{\partial\left(\rho u_{j} \omega\right)}{\partial x_{j}}=\frac{\gamma}{v_{t}} P-\beta \rho \omega^{2}+\frac{\partial}{\partial x_{j}}\left[\left(\mu+\sigma_{\omega} \mu_{t}\right) \frac{\partial \omega}{\partial x_{j}}\right]+2\left(1-F_{1}\right) \frac{\rho \sigma_{\omega 2}}{\omega} \frac{\partial k}{\partial x_{j}} \frac{\partial \omega}{\partial x_{j}}
$$

The two terms on the left side of Eqs. (2), (3) are related to the rate of change of $k$ or $\omega$ and the convective transport of $k$ or $\omega$, respectively. The three terms in common on the right side of Eqs. (2), (3) represent, in this order, the transport of $k$ or $\omega$ by turbulent diffusion, the rate of production of $k$ or $\omega$, and the rate of dissipation of $k$ or $\omega$. For the third term on the right side of Eq. 3,

Where:

$$
\begin{gathered}
P=\tau_{i j} \frac{\partial u_{i}}{\partial x_{j}} ; \tau_{i j}=\mu_{t}\left(2 S_{i j}-\frac{2}{3} \frac{\partial u_{k}}{\partial x_{k}} \delta_{i j}\right)-\frac{2}{3} \rho k \delta_{i j} ; S_{i j}=\frac{1}{2}\left(\frac{\partial u_{i}}{\partial x_{j}}+\frac{\partial u_{j}}{\partial x_{i}}\right) ; \mu_{t} \\
=\frac{\rho a_{1} k}{\max \left(a_{1} \omega, \Omega F_{2}\right)} ; \mu_{t}=\frac{\rho a_{1} k}{\max \left(a_{1} \omega, \Omega F_{2}\right)} \\
\phi=F_{1} \phi_{1}+\left(1-F_{1}\right) \phi_{2} ; F_{1}=\tanh \left(\arg _{1}^{4}\right) ; \arg _{1}=\min \left[\max \left(\frac{\sqrt{k}}{\beta^{*} \omega d}, \frac{500 v}{d^{2} \omega}\right), \frac{4 \rho \sigma_{\omega 2} k}{\mathrm{CD}_{k \omega} d^{2}}\right]
\end{gathered}
$$




$$
\begin{gathered}
\mathrm{CD}_{k \omega}=\max \left(2 \rho \sigma_{\omega 2} \frac{1}{\omega} \frac{\partial k}{\partial x_{j}} \frac{\partial \omega}{\partial x_{j}}, 10^{-20}\right) ; F_{2}=\tanh \left(\arg _{2}^{2}\right) ; \\
\arg _{2}=\max \left(2 \frac{\sqrt{k}}{\beta^{*} \omega d}, \frac{500 v}{d^{2} \omega}\right)
\end{gathered}
$$

$k-\Omega$ closure: $\sigma_{k 1}=0.85, \sigma_{w 1}=0.65, \beta_{1}=0.075$

$k$ - $\varepsilon$ closure: $\sigma_{k 2}=1.00, \sigma_{w 2}=0.856, \beta_{2}=0.0828$

The $\beta^{*}$ value can directly calibrate it, affecting how turbulence grows or dissipates. The final term in the $\omega$ equation (Eq. 3 ) represents the cross-diffusion that occurs as a result of the transformation of the $\varepsilon$ equation into an equation for $\omega$.

SST closure constants: $\beta^{*}=0.09, a_{1}=0.31$

\section{Numerical model}

\subsection{Solver and turbulence model}

The simulation that has been carried out was carried out with a Cartesian coordinate for numerical simulation. The operating conditions were set to be transient and iterative methods to reduce the margin of error by convergence. Water properties are used along with pressure and mass fluxes for the momentum equations. The velocity kept constant PRESTO (pressure staggered option) is highly recommended for fluid swirling characteristics inside the body. For turbulence, Reynolds stress (Shear Stress Transport model) and a Volume of Fluid (VOF) model were selected to evaluate; Semi implicit pressure linked equations combine the momentum and continuity laws to obtain derivatives of the pressure. The simulations are run with a fixed time step of $5.0 \times 10^{-4} \mathrm{~s}$. Furthermore, the effects of the controlled variables and parameters are discussed to validate experimental results. A velocity boundary condition is employed on the feed and pressure outlet boundary conditions for both the overflow and underflow, with an air backflow volume percentage of 1.0. When the air backflow volume fraction is set to 1.0, the simulation can build an air-core by drawing air via the overflow and underflow apertures when the swirl produces an area of negative pressure along the axis [13].

\section{Model validation}

At this reporting time, the lack of experimental data for the current study varied vortex finder lengths is only validated using similar CFD studies in the literature. Ghodrat et al [14] used a two-fluid model to simulate multiphase flow and hydrocyclone performance, paying specific attention to the impacts of vortex finder diameter, length, and shape over a wide range of feed solids concentrations. We selected this literature as it had the same numerical approach as we have undertaken. However, the size of the hydrocyclone is from the $d 75$. We had to continue with a $d 50$ hydrocyclone as our objective for this study. Overall, Figure 3 shows the trends obtained in the numerical conducted using the LES are in good agreement with SST model. 


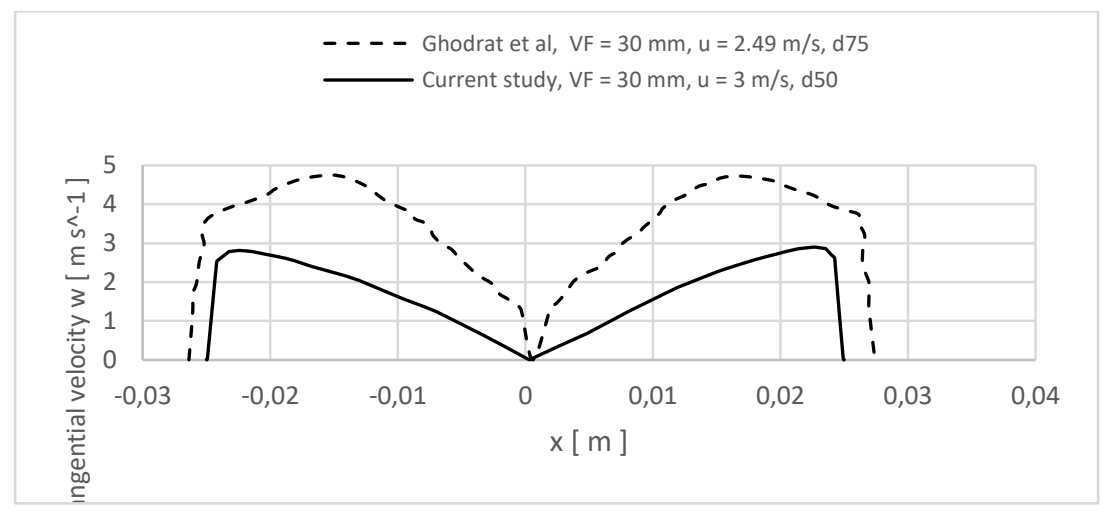

Fig. 3. Radial distribution of the tangential velocities in the horizontal plane located at $170 \mathrm{~mm}$ away from the underflow of the hydrocyclone Ghodrat et al [14].

The use of the VOF approach facilitated with the Large Eddy Simulation (LES) model should be used [14]. As it can produce results that are better comparable to experimental measurements should be used. Our observation is that LES model can better predict the tangential velocities. The trends the two approached area provide an expected velocity profile of particles experiencing centrifugal forces pushing against the wall. Our limit is also extenuated by much finer meshes that the LES model would require than the SST model. It is then computationally much more demanding at this moment. The transition to the LES model will be complimented by the acquisition of commercially available licence, using a cluster for high performance computing and conducting experiments.

\section{Results and discussion}

\subsection{Effects of vortex finder depth on pressure drop}

The vortex finder's primary function is to minimise any short circuit flow at the feed of the hydrocyclone [2]. In practice, only the difference between the inlet and the overflow pressure of the hydrocyclone is considered to calculate a pressure drop in a hydrocycle. As seen from the topology diagrams, the $35 \mathrm{~mm}$ depth hydrocyclone produces a lot of overflow pressure. Relative to the $30 \mathrm{~mm}$ depth hydrocyclone. This means that the pressure drop will be higher for the $\mathrm{VF}=35 \mathrm{~mm}$ cyclone; this will significantly impact the feed separation of the cyclone.

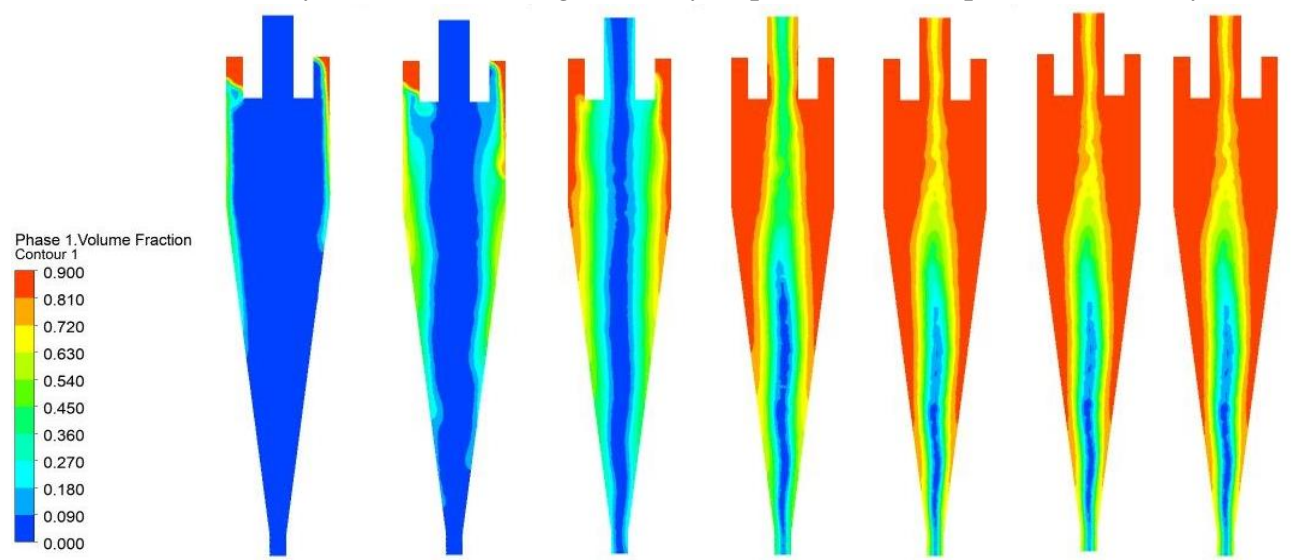

Fig. 4. Water's (Phase 1) forming process and the nature of the air core of $20 \mathrm{~mm}$ vortex finder over time: $t=0.05$ seconds to $t=4.05$ seconds viewed from left to right, at gradual time increments. 
The separation of the centrifugal swirl in the cyclone's core causes the vortex to become narrower, and the friction will increase. This can be seen in Figures 4 and 5 that the walls of the $35 \mathrm{~mm}$ hydrocyclone have a higher pressure drop. This influence on geometrical specifications on the pressure drop shows that the pressure drop is a function of the vortex finder depth. With a correct aspect ratio of the inlet/overflow diameter and vortex finder depth, a more acceptable feed separation can be produced. The separation efficiency of a hydrocyclone depends on the vortex finder depth as the particles are guided by the outside wall in the presence of the vortex finder. The particle re-entrainment duration in the underflow stream increases as the vortex finder depth increases, increasing separation efficiency [6]. Under a higher pressure, fine particles more easily moved towards the centre, which was favourable for the separation of fine particles. The particles were separated more efficiently due to high centrifugal force, and the swirling motion within the hydrocyclone increased as the feed velocity increased. The intensity of the centrifugal force is directly proportional to the tangential velocity. The main driving force in two-phase separation is centrifugal force [11].

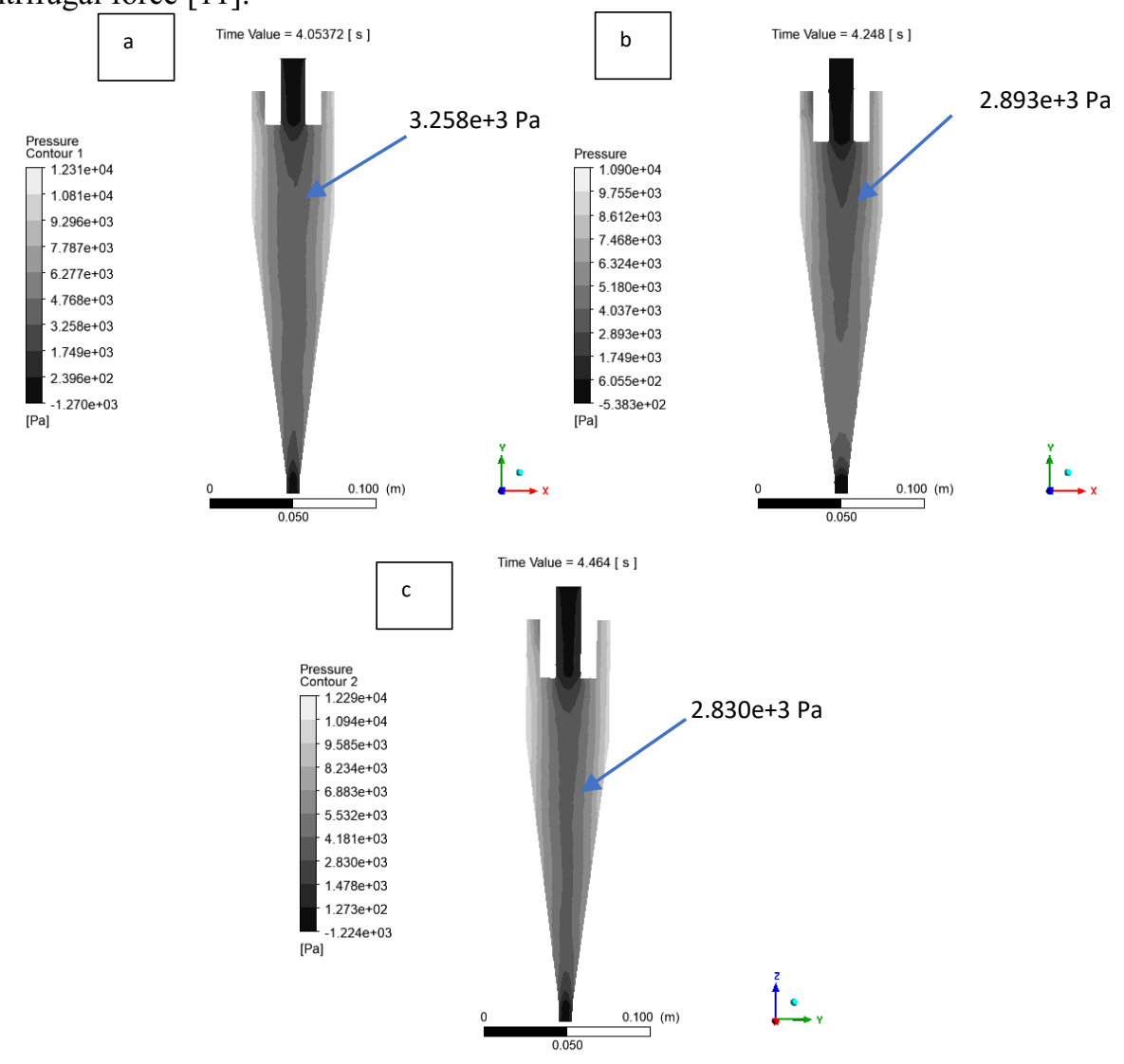

Fig. 5. Pressure gradient of the vortex finders in relation to the pressure drop across for (a) VF $=20$ $\mathrm{mm}$, (b) $\mathrm{VF}=30 \mathrm{~mm}$, (c) $\mathrm{VF}=35 \mathrm{~mm}$.

\subsection{Effect of vortex finder depth on the air core}

The prediction of the air-core profile is crucial for the flow dynamics to be described. The water is pushed against the wall by the tangential velocity, forming the air-core. As a result, 
as the tangential velocity decreases, the diameter of the air-core decreases [15]. It is seen in Figure 6 that the increasing the vortex finder length has a bearing in the magnitude and behaviour of the kinetic energy of the hydrocyclone. The tangential velocity increases with the VF length thus providing a better classification of the particles.

The axial velocity determines the particle retention time and split ratio in the hydrocyclone. At a lower axial velocity, the particles lingered in the hydrocyclone for longer and separated more thoroughly [11]. Narasimha et al [16] found that increasing the feed flow rate causes an increase in the maximum tangential velocity (accounting for the increase of kinetic energy) in a given cyclone, which in turn causes a decrease of the pressure at the hydrocyclone axis near the apex. If the pressure is lowered near the apex, it is easier for the air to be sucked in through the apex. Hence the air-core diameter increases with inlet flow rate.

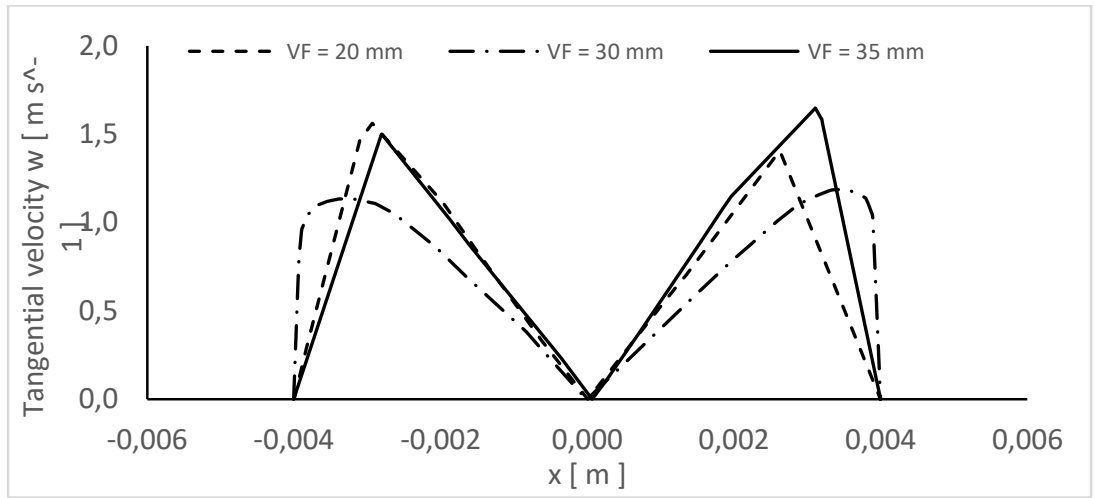

Fig. 6. The tangential velocity in the $y=0 \mathrm{~m}$ (underflow area) radial position.

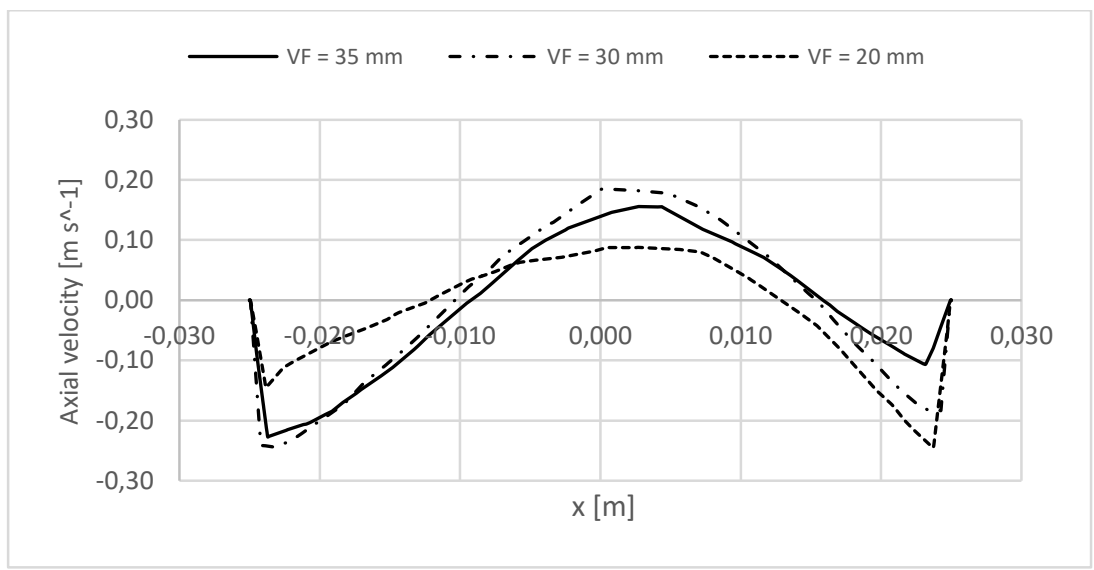

Fig. 7. The axial velocity in the $\mathrm{y}=0.169 \mathrm{~m}$ (diameter line, $d 50 \mathrm{~mm}$ ) radial position.

Neesse and Dueck [17] established an empirical formula (Eq. 4) to determine an air core diameter based on the radial pressure distribution in the hydrocyclone. The Navier-Stokes equations were used by Neesse and Dueck [17] to calculate the radius of an air core, assuming force equilibrium between pressure gradient and centrifugal force at the liquid-gas interface. This physical consideration leads to Eq. 4, which shows that the hydrocyclone shape is the primary determinant of the air core radius. 
$\frac{R_{\mathrm{a}}}{R_{\mathrm{c}}}=\left[1+\frac{2 n}{k_{w}^{2}}\left(\frac{R_{\mathrm{C}}}{R_{\mathrm{in}}}\right)^{2}\left(\frac{R_{\mathrm{in}}}{R_{\mathrm{o}}}\right)^{2 m}\left(\frac{p_{\text {in }}-p_{u}}{p_{\mathrm{in}}-p_{\mathrm{o}}}\right)\right]^{-1 / 2 n}$

Where: dimensionless relation for the air core radius $R_{a}$ as function of hydrocyclone radius $R_{c}$, inlet radius $R_{i n}$, overflow radius $R_{o}$ and the pressures in the underflow $p_{u}$ and in the overflow $p_{o}$. The values of $k=0.27, n=0.8$ and $m=1$ the empirical constants that depend on hydrocyclone geometry.

We used Eq. 4 to determine an air core diameter for a varied vortex finders. Presented in Figures 8 and 9 are the two extreme cases of our study. It is proving to be difficult to have air core formation that comes from instabilities of the multiphase flow up to the stable air core. The air diameter is still not stable along the centre hydrocyclone and the influence of the length of vortex finder as it increases the pressure drop in the overflow. It is not providing a stable air core. The air core diameter in the underflow over time is presented in figures Fig 8 and 9. It stabilizes from 1 second at a diameter of around $7.84 \mathrm{~mm}$ and $7.216 \mathrm{~mm}$ for our two extreme cases of VF of $20 \mathrm{~mm}$ and $35 \mathrm{~mm}$, respectively.
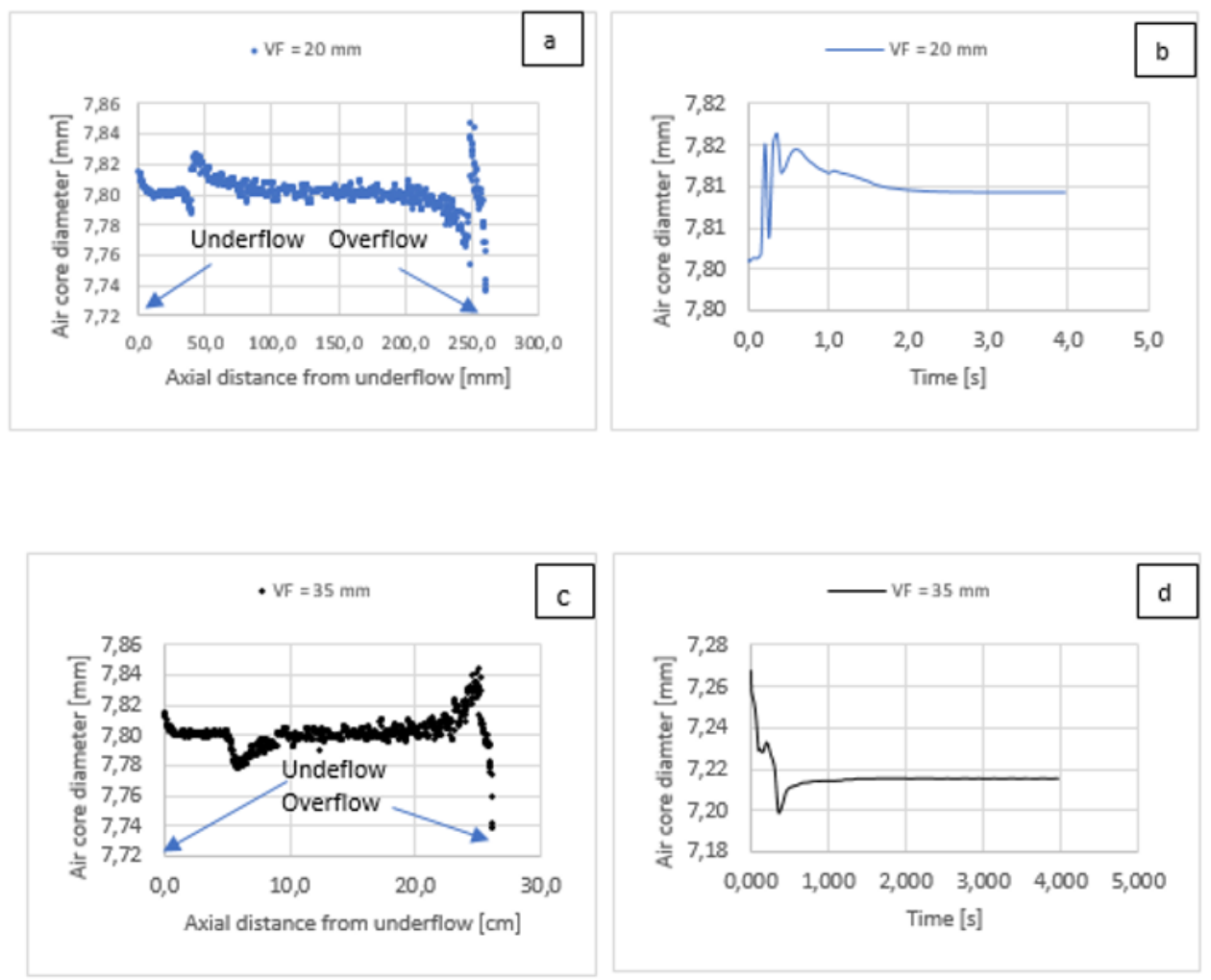

Fig. 8: (a, b) The air core along the axial distance at time 3.95 seconds (c, d) the air core stabilizing with time in the underflow region of the hydrocyclone with VF of $20 \mathrm{~mm}$ and $35 \mathrm{~mm}$, respectively. 

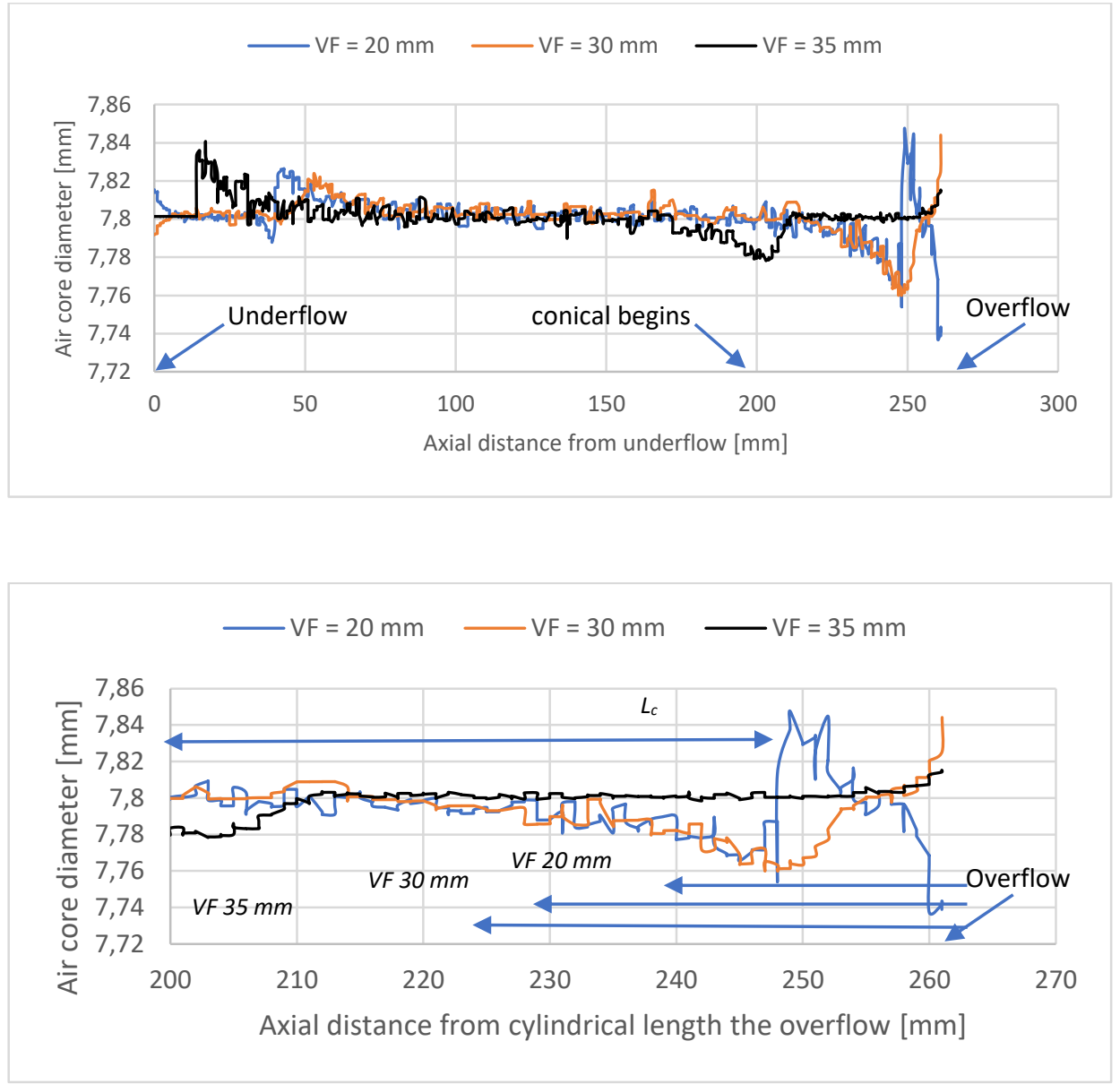

Fig. 9: Illustration of the positions of the $L_{c}$, cylindrical part length and the different vortex finders.

If the VF becomes longer and reaches the conical section, the current turbulence may force coarser particles to depart through the overflow stream rather than the intended underflow stream [2]. Our study of the VF $30 \mathrm{~mm}$ shows an almost constant air core diameter with the cylindrical part length but starts to quickly destabilize under $210 \mathrm{~mm}$ or closer to the cylindrical length that ends $200 \mathrm{~mm}$ from the overflow. Hence, there is a greater importance to have critical length of a VF to the cylindrical length. It is prevalent in maintaining a stable air core diameter. A suitable VF like the $35 \mathrm{~mm}$, as noticed in this study, proves a constant air flow through the core that leads the air to flow upwards to the overflow. The centrifugal force in the overflow pipe is minimized, preventing the formation of a stable air core. It is important to have the balance between making use of the advantages a longer VF provides like a stable air core, to the point or conical section. For the VF $35 \mathrm{~mm}$, the air core diameter decreases and remains stable with increasing VF length. The change is more obvious for a longer vortex finder length, and this may be due to the strengthening tangential velocity and increased pressure drop [18]. However, extending the vortex finder shortens the natural vortex in the cyclone body, reducing the chance of small particles separating from the vortex [19]. 


\section{Conclusion}

An optimization numerical study using the SST model was conducted for the varied vortex finders of a $d 50$ hydrocyclone. We find that the designs of new geometries opens a new chapter in the optimization of hydrocyclones. We have computed pressure drop is lower at vortex finders with a shorter length. The length can be increased to match the pressure drop of the standard hydrocyclone. The air core diameter decreases and remains stable with increasing under the conical section. As a result, the flow field characteristics inside the hydrocyclone without an air core become more separation-friendly. The airflow as characteristics of the air core may be used to control the hydrocyclone.

\section{Acknowledgement}

The author would like to thank supervisor Thokozani Justin Kunene and co-supervisor Lagouge Kwanda Tartibu and for the support guidance patience during the preparation of this paper. The institution University of Johannesburg for financially committing to the conference cost.

\section{REFERENCES}

[1] B. Cui, C. Zhang, D. Wei, S. Lu, and Y. Feng, "Effects of feed size distribution on separation performance of hydrocyclones with different vortex finder diameters," Powder Technol., vol. 322, pp. 114-123, 2017.

[2] L. F. Martínez, A. G. Lavín, M. M. Mahamud, and J. L. Bueno, "Vortex finder optimum length in hydrocyclone separation," Chem. Eng. Process. Process Intensif., vol. 47, no. 2, pp. 192-199, 2008.

[3] K. Heiskanen, "Experimental hydrocyclone roping models," Chem. Eng. J., vol. 80, no. 1-3, pp. 289-293, 2000.

[4] T. Dyakowski and R. A. Williams, "Prediction of air-core size and shape in a hydrocyclone," J. Miner. Process, vol. 43, pp. 1-14, 1995.

[5] M. Narasimha, M. S. Brennan, and P. N. Holtham, "CFD modeling of hydrocyclones: Prediction of particle size segregation," Miner. Eng., vol. 39, pp. 173-183, 2012.

[6] G. Patra, S. Chakraborty, and B. C. Meikap, "Role of vortex finder depth on pressure drop and performance efficiency in a ribbed hydrocyclone," South African J. Chem. Eng., vol. 25, pp. 103-109, 2018.

[7] T. Braun and M. Bohnet, "Influence of feed solids concentration on the performance of hydrocyclones," Chem. Eng. Technol., vol. 13, no. 1, pp. 15-20, 1990.

[8] R. Raesi and R. Maddahian, "Numerical investigation of air-injected deoiling hydrocyclones using population balance model," Chem. Eng. Sci., vol. 248, p. $117103,2021$. 
[9] R. Sripriya, M. D. Kaulaskar, S. Chakraborty, and B. C. Meikap, "Studies on the performance of a hydrocyclone and modeling for flow characterization in presence and absence of air core," Chem. Eng. Sci., vol. 62, no. 22, pp. 6391-6402, 2007.

[10] M. A. Hararah, E. Endres, J. Dueck, L. Minkov, and T. Neesse, "Flow conditions in the air core of the hydrocyclone," Miner. Eng., vol. 23, pp. 295-300, 2010.

[11] F. Li et al., "Numerical Analysis on the Effect of Combined-Curve Tapered Segment on the Flow Field and Separation Performance of Hydrocyclones," Arab. J. Sci. Eng., vol. 172, no. August, 2021.

[12] F. R. Menter, "Two-equation eddy-viscosity turbulence models for engineering applications," AIAA J., vol. 32, no. 8, pp. 1598-1605, 1994.

[13] M. Narasimha, M. Brennan, and \& P. N. Holtham, "A Review of CFD Modelling for Performance Predictions of Hydrocyclone," Eng. Appl. Comput. Fluid Mech., vol. 1, no. 2, pp. 109-125, 2007.

[14] M. Ghodrat, S. B. Kuang, A. B. Yu, A. Vince, G. D. Barnett, and P. J. Barnett, "Numerical analysis of hydrocyclones with different vortex finder configurations," Miner. Eng., vol. 63, pp. 125-138, 2014.

[15] J. A. Delgadillo and R. K. Rajamani, "Exploration of hydrocyclone designs using computational fluid dynamics," International Journal of Mineral Processing, vol. 84, no. 1-4. pp. 252-261, 2007.

[16] M. Narasimha, M. Brennan, and P. N. Holtham, "Large eddy simulation of hydrocyclone - prediction of air-core diameter and shape," vol. 80, pp. 1-14, 2006.

[17] T. Neesse and J. Dueck, "Air core formation in the hydrocyclone," Miner. Eng., vol. 20, no. 4, pp. 349-354, 2007.

[18] B. Tang, Y. Xu, X. Song, Z. Sun, and J. Yu, "Numerical study on the relationship between high sharpness and configurations of the vortex finder of a hydrocyclone by central composite design," Chem. Eng. J., vol. 278, pp. 504-516, 2015.

[19] F. Ficici, A. Vedat, and M. Kapsiz, "The effects of vortex finder on the pressure drop in cyclone separators," Int. J. Phys. Sci., vol. 5, no. 6, pp. 804-813, 2010. 